\title{
Influencia de las emociones en las habilidades emprendedoras de los propietarios de servicios turísticos de la ciudad de Puyo
}

\section{Influence of emotions on the entrepreneurial skills of the owners of tourist services in the city of Puyo}

\author{
Luis Oswaldo Manosalvas Vaca ${ }^{1 *}$, Génesis Andrea Chávez Brito ${ }^{1}$, Daniela Yojaira Guevara Rueda ${ }^{1}$ y Carlos \\ Anibal Manosalvas Vaca ${ }^{1}$ \\ ${ }^{1}$ Universidad Estatal Amazónica \\ *lmanosalvas@uea.edu.ec
}

DOI: https://doi.org/10.26871/killkana_social.v2i3.327

\begin{abstract}
Resumen
El emprendimiento es una variable determinante en el desarrollo social, económico y humano de los países y regiones, según los resultados reportados en las investigaciones científicas sobre emprendimiento, existen varios factores que generan influencia sobre el comportamiento emprendedor, entre las características que más han generado interés en el campo científico empresarial son los aspectos personales cognitivos y emocionales que direccionan el comportamiento empresarial, por lo tanto, la presente investigación analiza la relación de las emociones en las habilidades emprendedoras en los propietarios de los diferentes servicios turísticos de la ciudad de Puyo, considerando, que la mencionada ciudad se encuentra en un lugar estratégico de la Amazonía ecuatoriana y que permite el desarrollo empresarial de su región, en especial del sector turístico. Para el desarrollo de este estudio se utilizó una metodología de investigación, cuantitativa, de carácter descriptivo y con un horizonte transversal, se aplicaron técnicas estadísticas descriptivas, inferenciales y multivariantes por medio de un análisis factorial confirmatorio y una relación de ecuaciones estructurales MES. Los resultados demostraron que la Inteligencia Emocional y las dimensiones que miden las emociones tiene influencia significativa con la Autoeficacia Emprendedora, variable que mide la creencia en las propias habilidades emprendedoras, por lo tanto, se evidencia que las creencias en las propias habilidades emprendedoras de los empresarios del sector turístico se relacionan de manera directa y significativa con el manejo de las emociones que poseen. Estos resultados permiten contribuir en el desarrollo personal de los emprendedores de la ciudad de Puyo y de la misma manera, permitirá favorecer el bienestar emocional y desempeño laboral de los actores involucrados en los emprendimientos turísticos.
\end{abstract}

Palabras clave: Inteligencia Emocional, Autoeficacia Emprendedora, Habilidades emprendedoras.

\begin{abstract}
Entrepreneurship is a determining variable in the social, economic and human development of countries and regions, according to the results reported in scientific research on entrepreneurship, there are several factors that generate influence on entrepreneurial behavior, among the characteristics that have generated most interest in the scientific business field are the personal cognitive and emotional aspects that guide business behavior, therefore, this research analyzes the relationship of emotions in entrepreneurial skills in the owners of the different tourist services of the Puyo city, considering , Puyo is located in a strategic place in the Ecuadorian Amazon and that it allows the business development of its region, especially in the tourism sector. For the development of this study we used a quantitative, descriptive research methodology with a transversal, descriptive, inferential and multivariate statistical techniques were applied by means of a confirmatory factorial analysis and a relation of MES structural equations. The results showed that the Emotional Intelligence and the dimensions that measure the emotions have a significant influence with the Entrepreneurial Self-Efficacy, a variable that measures the entrepreneurial skills, therefore, it is evident that the beliefs in the entrepreneurial skills of the entrepreneurs in the tourism sector relate directly and significantly to the management of the emotions they possess. These results allow to contribute in the personal development of the entrepreneurs of Puyo and in the same way, it will allow to favor the emotional well-being and labor performance of the actors involved in the tourism ventures.
\end{abstract}

Key words: Emotional Intelligence, Entrepreneurial Self-Efficacy, Entrepreneurial Skills. 


\section{Introducción}

El crecimiento económico se evidencia por parte de los diferentes emprendimientos que existen, sus cambios e innovaciones ayudan para la competitividad existente en el mercado. El turismo es una actividad dinámica, generadora de recursos económicos de los países y específicamente en el Ecuador, permitiendo al turista extranjero y nacional conocer la diversidad que posee el país (Caiza y Molina, 2012). En la actualidad, el turismo es una fuente que incentiva a la inversión, es por ello, que existe un incremento de emprendimiento en el sector turístico, pero pueden verse afectados por diferentes factores de tipo conductual, financiero, ambiental y político por lo que es importante considerar las decisiones que se tomen al respecto (Lado, Vivel, Otero, y Neira, 2015). En el año 2016, según el Global Entrepreneurship Monitor (GEM) indica que el Ecuador es el país con más actividad emprendedora de la región latinoamericana, situándose entre los primeros lugares de los países que se realiza el análisis, sin embargo, en cuanto a la creación de empleo, los negocios ecuatorianos son muy bajos, tanto así que en los próximos años no se espera generar plazas de trabajo, lo que demuestra también un estancamiento en los negocios que atraigan a clientes internacionales, comparándolos con Colombia y Chile que poseen una alta demanda (Lasio, Caicedo, Ordeñana, y Samaniego, 2016). Hoy en día las empresas necesitan que el personal que labora con ellos sean líderes, ya que su comportamiento para comprender y manejar las habilidades es fundamental para la toma de decisiones. La comunicación y el desarrollo del personal ayuda a cumplir con los objetivos planteados. Entre las características más relevantes que se ha encontrado de los factores que influye en el comportamiento emprendedor, es el perfil emocional (Manosalvas, 2017). Una persona puede pensar, sentir y de estos dos procesos distintos trabajar eficazmente, es por ello la importancia de la Inteligencia Emocional como un factor clave dentro de las empresas, ya que un líder es capaz de motivarse y mantenerse frente a las desilusiones, reconocer los estados emocionales de su personal y crear experiencias emocionales considerables para los resultados esperados dentro de su organización (Diez \& Sanchez, 2017). Un emprendedor emocionalmente inteligente es capaz de resolver problemas, analizar su entorno y alcanzar el éxito (Salvador, 2008). Gracias a los beneficios que ha tenido la Inteligencia Emocional en muchas empresas para ayudar al liderazgo, trabajo en equipo, mejora del servicio, y motivación personal (Avila, Sandoval, \& Tejeda, 2008). Se ha considerado realizar un análisis en los emprendedores de servicios turísticos de la ciudad de Puyo, utilizando la metodología de Inteligencia Emocional propuesta por Mayer y Salovey en el año de 1990 para determinar si existe influencia sobre las habilidades en el comportamiento emprendedor en el sector turístico.

Pastaza es la provincia más extensa del Ecuador y posee una gran riqueza cultural, natural y gastronómica, con un gran abanico de servicios turísticos en el área de restauración, tour operadores, y alojamiento de todo tipo de categorías, haciendo de este un lugar placentero para todo aquel que lo visita (Silva y Alban, 2017).

Según la CAPTUR PASTAZA (Cámara de Turismo de la Provincia de Pastaza) han indicado que existe desinterés por parte de las autoridades del GAD descentralizado y el Ministerio de Turismo en la promoción y seguimiento del sector turístico de la ciudad. El catastro turístico del año 2016 de la ciudad de Puyo, indica que existen 46 emprendimientos de alojamiento, 14 parques de atracciones estables, 168 emprendimientos de Alimentos y Bebidas, 10 establecimientos de Operación e Intermediación y 2 de Transporte turístico, inscritos en el Ministerio de Turismo y 15 emprendimientos de alojamiento registrados solamente en la Intendencia de Policía, de la misma manera se puede evidenciar que la falta de interés por parte de las autoridades ha ocasionado que los emprendimientos en el sector turístico no tengan una buena aceptación en el mercado. Por esta razón, varios propietarios de establecimientos turísticos, han manifestado que tienen un escaso interés en generar inversión que pueda desarrollar sus emprendimientos en forma efectiva. Por lo tanto, se puede percibir que existe una afectación del área emocional, dando lugar a una baja autoestima por considerar que el servicio turístico no es rentable en la ciudad de Puyo, evidenciando, que no existe un interés por cumplir con los reglamentos y normativas establecidos por el Ministerio de Turismo, de la misma manera se observa que la promoción turística no se considera relevante, lo cual puede determinar como uno de los factores más importantes de la actividad emprendedora en el servicio turístico. Para el desarrollo de la investigación se ha planteado la siguiente pregunta: ¿Las habilidades emprendedoras de los propietarios de servicios turísticos de la ciudad de Puyo se encuentran influenciadas por sus emociones?, lo cual ha permitido generar la siguiente hipótesis: H1: Las emociones tienen una influencia significativa con las habilidades emprendedoras de los propietarios de servicios turísticos de la ciudad de Puyo.

\subsection{Emprendimiento y Emprendedor}

Es la actividad llevada a cabo por una persona denominada emprendedor, que persigue un fin económico, político, social, el cual posee características de innovación, siendo así el emprendimiento un conjunto de recursos que aprovecha las oportunidades que se presentan creando un gran valor en la empresa (Amorós, Etchebarne, y Felzensztein, 2012).

Las acciones emprendedoras se pueden realizar individualmente o formando parte de un grupo estructurado o no estructurado y se desarrolla en diversos ámbitos como el emprendimiento empresarial, en el cual el aspecto económico es la motivación principal, el emprendimiento social que busca, promueve y sostiene valores, recursos y capacidades al servicio del entorno social; emprendimiento publico fundamentado en la política que traslada la cultura empresarial al sector público, en los últimos años tanto en 
los ámbitos económicos como políticos se ha dado una idea creciente donde el emprendedor es un guía hacia un cambio y crecimiento óptimo de la economía. De esta manera, en los países se empezó a mejorar el entendimiento de la población sobre el papel de los emprendedores en la economía, dentro de esta misma línea alrededor del mundo los gobiernos mediante iniciativas fomentan el emprendimiento como una inversión para el crecimiento económico (Salinas y Osorio, 2017).

\subsection{Emprendimiento en el sector turístico}

El turismo es una actividad económica, social y cultural que se ve influenciado por el desplazamiento de las personas a diferentes lugares fuera de su residencia habitual por diferentes motivos. El turismo genera impactos sobre la actividad económica y en los lugares que son visitados, todo debido a la oferta y demanda que existe por parte de los bienes y servicios. El crecimiento económico que ha tenido el turismo, lo ha convertido en el sector con mayor éxito. Por lo que es el motor clave del progreso socio económico, actor del comercio internacional y principal fuente de ingreso (Organizacion Mundial de Turismo, 2007).

El turismo es una de las actividades que impacta al sector económico y sus negocios son el resultado, gracias a las inversiones realizadas por cada sector. El aumento de la calidad de vida y el aprovechamiento de los recursos ha tenido avances significativos en la industria turística, generando empleo y crecimiento económico (Gessa y Toledano, 2011). Pastaza, es una provincia comercial, y su aporte a la industria turística depende del incentivo por parte de cada propietario y autoridades ya que es la alternativa del futuro para el desarrollo del sector turístico, si se trabaja en conjunto con el gobierno, el sector privado y las comunidades (Padilla, Quispe, y Jimenez, 2016).

\subsection{Comportamiento y habilidades de un emprendedor}

Es posible que el comportamiento del emprendedor influya en el éxito de un emprendimiento y son diferentes los factores en su entorno que llevan a explotar su potencial, generando como resultado que las decisiones tengan éxito o fracaso. Las habilidades generalmente son aplicadas en ideas viejas para generar oportunidades de negocios, pero muchas de las empresas se resisten a los cambios. Es así como países latinoamericanos se inclinan más al extremo administrativo del comportamiento, que al extremo emprendedor por carencia de recursos y no aprovechan las oportunidades presentadas (Dehter, 2010).

La Autoeficacia Emprendedora está determinada por algunas características intrínsecas, es decir que es importante lo que cree cada emprendedor de sí mismo, de sus capacidades para tener éxito en la creación de su emprendimiento (Salvador, 2008). Las principales dimensiones que debería desarrollar un emprendedor para tener éxito en su propia empresa, resaltando las siguientes dimensiones: Afrontar retos inesperados, es la capacidad de trabajar cuando algo inesperado ocurre, desarrollando proyectos innovadores sin seguir procedimientos ni tareas que han sido establecidos para lograr esto se necesita que los directivos sean capaces de tolerar lo imprevisto y se adapten a los cambios. Construir un entorno innovador, en este factor se da énfasis a la capacidad de los líderes y al personal que se le conoce como mandos directivos para impulsar y apoyar la creatividad, innovación y responsabilidad de los trabajadores. Definir los objetivos principales, se debe crear proyectos con una visión y objetivos claros para que de esta manera se unan todos los colaboradores de una empresa y sean inspirados para hacer realidad la meta que se trazaron. Lanzar productos o servicios, este factor hace referencia a las habilidades que tienen los líderes y mandos medios para lanzar al mercado nuevos productos y servicios estableciendo nuevas vías de comercialización. Desarrollar productos y oportunidades de mercado, para los emprendedores tener la capacidad de crear nuevos productos para satisfacer las necesidades de los clientes es de suma importancia ya que el reconocimiento de oportunidades es una dimensión clave en la autoeficacia emprendedora. Construir y dirigir el equipo, es fundamental que los líderes y mandos medios sean capaces de mantener una buena relación con el personal e integrarles de una manera responsable y respetuosa para que se muestren colaboradores y centrados con el objetivo principal de la empresa (Moriano, Topa, Molero, Entenaza, y Levy, 2012).

\subsection{Inteligencia Emocional}

La Inteligencia Emocional nace a partir del trabajo de Mayer y Salovey en 1990, indica que es la habilidad de una persona en enfrentarse a la vida y cómo integra sus emociones con la realidad, para poder medir la Inteligencia Emocional se han creado herramientas metodológicas como el TMMS-48 (Duran, 2013). Nuestro potencial para entender y aprender habilidades es determinado por la inteligencia emocional, la misma que se compone de cinco elementos fundamentales: el conocimiento de uno mismo, empatía, autorregulación, motivación y destreza para las relaciones. En cuanto a la aptitud emocional muestra que nuestro potencial puede cambiar drásticamente de acuerdo a las facultades que aplicamos en el área laboral, en el caso de la empatía nuestra eficiencia será mejor a la hora de servir a los clientes. Igualmente, la confidencialidad está basada en la capacidad de manejar los impulsos y emociones correctamente en conjunto con la autorregulación. Una persona puede destacarse en su área de trabajo como en el servicio al cliente por la práctica de la confidencialidad, así mismo gracias a estas aptitudes emocionales una persona puede llegar a tener un papel importante en el liderazgo de su empresa, logrando que otros empleados ejecuten sus actividades con más efectividad. Las emociones son contagiosas, el estado de ánimo y el comportamiento de cada uno de nosotros influye drásticamente en los demás; influir negativa o positivamente en otras personas es algo totalmente natural, al contacto de dos seres humanos existe 
interacción que determina los sucesos que pueden ocurrir a continuación, las emociones son contagiosas todas estas capacidades se apoyan en el hecho primordial de que cada uno de nosotros influye en el estado de ánimo de los demás, nuestro estado de ánimo al igual que un virus social es captado por las demás personas, este intercambio emocional es parte de una invisible economía interpersonal que existe en toda interacción humana. (Ortega, 2014). Para el TMMS-48 existen tres dimensiones claves en la Inteligencia Emocional: Atención a los sentimientos, Claridad en cuanto a emociones y Reparación emocional (Extremera y Fernández, 2005).

\subsection{Relación entre la Inteligencia Emocional y Autoefi- cacia Emprendedora}

La Inteligencia Emocional es fundamental en la Autoeficacia Emprendedora ya que los emprendedores utilizan sus emociones para creer en la capacidad de sus habilidades, por lo tanto, tendrán mayor éxito en sus emprendimientos al momento de enfrentarse al entorno (Salvador, 2008). La relación que existe entre la Inteligencia Emocional y Autoeficacia Emprendedora es positiva porque las personas perciben de mejor manera sus oportunidades, influenciada en el comportamiento empresarial, ya que las personas con alta Inteligencia Emocional son tolerantes al estrés y creen en sus propias capacidades (Manosalvas, 2017).

La Inteligencia Emocional influye significativamente en las diferentes habilidades de un emprendedor, concretamente en la iniciativa, liderazgo, creatividad, la necesidad de logros, la tolerancia al cambio y también al manejo de problemas. El mayor impacto que se puede apreciar en los emprendedores es en el liderazgo, mejorando muy positivamente sus interacciones sociales, también se refleja la creatividad del individuo al momento de enfrentar los diversos cambios que pueden surgir en una organización o empresa, esta inteligencia modifica el entorno del emprendedor facilitándole comprender a los demás individuos, así como su comunicación empática con los demás. Así mismo esta se manifiesta de manera importante en sus vidas diarias, debido a que les ayuda a actuar, elegir, liderar y tomar decisiones de una forma eficiente. También ayuda a percibir emociones, a relacionarse con las demás personas, tener estas habilidades en conjunto permite actuar eficaz y eficientemente en las acciones y proyectos emprendidos, la Inteligencia Emocional va de la mano con la autoeficacia emprendedora, mejorando los proyectos emprendidos positiva y significativamente, de manera especial en cuanto al uso y la regulación de las emociones, debido a que tienen una relación directa con las habilidades empresariales las que ayudan a desarrollar las actividades emprendedoras (Maldonado, Castro, y Corrales, 2015). Los distintos resultados que se han obtenido, han permitido ver el gran impacto que provoca las emociones en el autoempleo, es por esto que se indica la posibilidad de realizar programas donde aprendan a mejorar y desarrollar habilidades para el control de las emociones poniendo énfasis y así tener profesionales capacitados para un futuro (Salvador, 2008).

\section{Método}

La metodología de investigación fue desarrollada mediante instrumentos de investigación validados y confiables que ayudaron a la obtención de información sobre los diferentes emprendimientos en servicios turísticos que tiene la ciudad de Puyo, el tipo de investigación es descriptivo por sus características que indican detalles específicos de los procesos que intervienen en el comportamiento emprendedor, además se aplicó un análisis multivariante para desarrollar el modelo de Análisis Factorial Confirmatorio y de Ecuaciones Estructurales, tiene un enfoque cuantitativo con un horizonte transversal que analiza factores emocionales y habilidades propias que posee un emprendedor.

Se aplicó el instrumento de medición específico de Autoeficacia Emprendedora en su versión adaptada y validada en España por Moriano et al. (2006), el cual mide la creencia de la persona en sus propias habilidades para desempeñar las tareas necesariamente requeridas para la creación de una nueva empresa, mediante una escala de 23 preguntas con respuesta en formato Likert de cinco categorías, que van desde 1 (Completamente incapaz) a 5 (Perfectamente capaz). La encuesta de Inteligencia Emocional en su adaptación Trait Meta-Mood Scale traducida al español, determina la capacidad de una persona para controlar sus emociones, y posee 48 preguntas con respuestas en formato Likert de 5 categorías, va desde 5 (Completamente de acuerdo) a 1(Completamente en desacuerdo). Se aplicaron los instrumentos de medición a 255 emprendedores del sector turístico de la ciudad de Puyo, en los sectores de alojamiento, restauración, operadores de turismo y otros.

\section{Análisis de Resultados}

\subsection{Análisis descriptivo}

Tabla 1 Género de la muestra

\begin{tabular}{|cc|c|c|c|c|}
\hline & & Frecuencia & Porcentaje & $\begin{array}{c}\text { Porcentaje } \\
\text { válido }\end{array}$ & $\begin{array}{c}\text { Porcentaje } \\
\text { acumula- } \\
\text { do }\end{array}$ \\
\hline Válido & Hombre & 98 & 38,4 & 38,4 & 38,4 \\
& Mujer & 157 & 61,6 & 61,6 & 100,0 \\
& Total & 255 & 100,0 & 100,0 & \\
\hline
\end{tabular}

Tabla 2 Nivel de Estudio de la muestra

\begin{tabular}{|c|c|c|c|c|}
\hline & Frecuencia & Porcentaje & $\begin{array}{c}\text { Porcentaje } \\
\text { válido }\end{array}$ & $\begin{array}{c}\text { Porcentaje } \\
\text { acumula- } \\
\text { do }\end{array}$ \\
\hline Primaria & 16 & 6,3 & 6,3 & 6,3 \\
Bachillerato & 122 & 47,8 & 47,8 & 54,1 \\
Universidad & 93 & 36,5 & 36,5 & 90,6 \\
Otros & 24 & 9,4 & 9,4 & 100,0 \\
Total & 255 & 100,0 & 100,0 & \\
\hline
\end{tabular}


Tabla 3 Ganancia económica de los emprendimientos

\begin{tabular}{|cc|c|c|c|c|}
\hline & Frecuencia & Porcentaje & $\begin{array}{c}\text { Porcentaje } \\
\text { válido }\end{array}$ & $\begin{array}{c}\text { Porcentaje } \\
\text { acumula- } \\
\text { do }\end{array}$ \\
\hline Válido & Hasta 500 & 63 & 24,7 & 24,7 & 24,7 \\
& $500-1000$ & 106 & 41,6 & 41,6 & 66,3 \\
& $1000-2000$ & 73 & 28,6 & 28,6 & 94,9 \\
& $2000-4000$ & 13 & 5,1 & 5,1 & 100,0 \\
& Total & 255 & 100,0 & 100,0 & \\
\hline \multicolumn{7}{|c|}{}
\end{tabular}

\subsection{Análisis de confiabilidad}

Tabla 4 Confiabilidad de las Escalas

\begin{tabular}{|c|c|c|c|}
\hline Constructo & Subconstructos & $\begin{array}{l}\text { Alpha de } \\
\text { Cronbach }\end{array}$ & $\begin{array}{l}\text { Número } \\
\text { de Ítems }\end{array}$ \\
\hline \multirow[t]{4}{*}{$\begin{array}{c}\text { Inteligencia } \\
\text { Emocional }\end{array}$} & & 0.958 & 48 \\
\hline & $\begin{array}{l}\text { Atención } \\
\text { Emocional }\end{array}$ & 0.912 & 21 \\
\hline & $\begin{array}{c}\text { Claridad } \\
\text { Emocional }\end{array}$ & 0.863 & 15 \\
\hline & $\begin{array}{l}\text { Reparación } \\
\text { Emocional }\end{array}$ & 0.848 & 12 \\
\hline \multirow[t]{7}{*}{$\begin{array}{c}\text { Autoeficacia } \\
\text { Emprendedora }\end{array}$} & & 0.961 & 23 \\
\hline & $\begin{array}{c}\text { Afrontar } \\
\text { cambios } \\
\text { Inesperados }\end{array}$ & 0.814 & 3 \\
\hline & $\begin{array}{l}\text { Construir un } \\
\text { entorno } \\
\text { innovador }\end{array}$ & 0.854 & 4 \\
\hline & $\begin{array}{c}\text { Definir el } \\
\text { objetivo } \\
\text { central del } \\
\text { negocio }\end{array}$ & 0.706 & 3 \\
\hline & $\begin{array}{c}\text { Desarrollar los } \\
\text { recursos } \\
\text { humano clave }\end{array}$ & 0.754 & 3 \\
\hline & $\begin{array}{c}\text { Descubrir } \\
\text { nuevos } \\
\text { productos y } \\
\text { oportunidades } \\
\text { de mercado }\end{array}$ & 0.885 & 7 \\
\hline & $\begin{array}{c}\text { Iniciar } \\
\text { relaciones con } \\
\text { inversores }\end{array}$ & 0.755 & 3 \\
\hline
\end{tabular}

El resultado de la tabla muestra que todas las escalas superan el valor mínimo de 0.7 por lo que se puede concluir que las escalas son confiables.

\subsection{Evaluación de Bondad de Ajuste}

Tabla 5 Índices de Ajuste del Modelo de Inteligencia emocional

\begin{tabular}{ccc}
\hline Índice & Valor & $\begin{array}{c}\text { Nivel de } \\
\text { Aceptación }\end{array}$ \\
\hline CFI & 0.949 & $\geq 0,95$ \\
RMSEA & 0.045 & $\leq 0,05$ \\
GFI & 0.892 & $>0.90$ \\
AGFI & 0.793 & $>0.80$
\end{tabular}

Tabla 6 Índices de Ajuste del Modelo Autoeficacia Emprendedora

\begin{tabular}{ccc}
\hline Índice & Valor & $\begin{array}{c}\text { Nivel de } \\
\text { Aceptación }\end{array}$ \\
\hline CFI & 0.975 & $\geq 0,95$ \\
RMSEA & 0.051 & $\leq 0,05$ \\
GFI & 0.923 & $>0.90$ \\
AGFI & 0.812 & $>0.80$ \\
\hline
\end{tabular}

Se puede indicar que los datos obtenidos en ambos instrumentos de medición se ajustan de manera adecuada al modelo estadístico planteado.

\subsection{Validez Convergente y Discriminante de los Modelos}

La validez convergente evalúa si los constructos con sus preguntas miden lo que tienen que medir de manera significativa y la validez discriminante indica que cada constructo debe medir en forma diferente (Chion y Vincent, 2016). Todos los estimados de cada ítem hacía los subconstructos son significativos ( $\mathrm{p}$-valor $<0.05$ ), por lo tanto, se indica que existe validez convergente (Anderson y Gerbing, 1988). La validez discriminante, que es la verificación de que no exista correlación entre los constructos, señaló que todas las diferencias son significativas, por lo tanto, se puede evidenciar que los instrumentos de medición tienen dimensiones que miden de forma diferente (Chion y Vincent, 2016).

\subsection{Modelo de Ecuaciones estructurales}

Se calculó el coeficiente de regresión en las relaciones de las variables de estudio para comprobar el enunciado de la hipótesis, se pudo demostrar que existe una relación significativa. La relación entre Inteligencia emocional y Autoeficacia emprendedora (tomando este último como un modelo de segundo orden donde los subconstructos dependen del gran constructo autoeficacia emprendedora) es de $\mathrm{r}$ $=0.308$ con $\mathrm{p}$-valor $<0.05$.

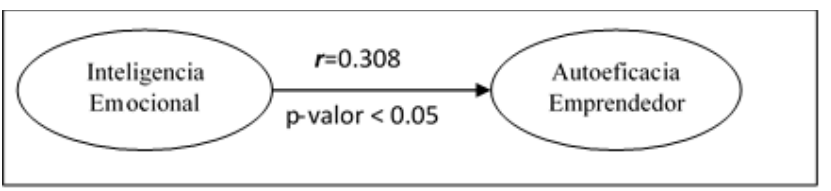

Figura 1. Modelo de Ecuaciones Estructurales 
Tabla 7 Relación entre Inteligencia Emocional y dimensiones de Autoeficacia Emprendedora

\begin{tabular}{|c|c|c|c|c|}
\hline & & & Estimado & p-valor \\
\hline $\begin{array}{c}\text { Afrontar cambios } \\
\text { inesperados }\end{array}$ & $<-$ & $\begin{array}{c}\text { I } \\
\text { Emocional }\end{array}$ & 0,666 & $* * *$ \\
\hline $\begin{array}{c}\text { Construir un entorno } \\
\text { innovador }\end{array}$ & $<-$ & $\begin{array}{c}\text { I } \\
\text { Emocional }\end{array}$ & 0,720 & $* * *$ \\
\hline $\begin{array}{c}\text { Definir el objetivo } \\
\text { central del negocio }\end{array}$ & $<-$ & $\begin{array}{c}\text { I } \\
\text { Emocional }\end{array}$ & 0,714 & $* * *$ \\
\hline $\begin{array}{c}\text { Desarrollar los recursos } \\
\text { humano clave }\end{array}$ & $<-$ & $\begin{array}{c}\text { I } \\
\text { Emocional }\end{array}$ & 0,518 & $* * *$ \\
\hline $\begin{array}{c}\text { Descubrir nuevos } \\
\text { productos y } \\
\text { oportunidades de } \\
\text { mercado }\end{array}$ & $<-$ & $\begin{array}{c}\text { I } \\
\text { Emocional }\end{array}$ & 0,604 & $* * *$ \\
\hline $\begin{array}{c}\text { Iniciar relación con } \\
\text { inversores }\end{array}$ & $<-$ & $\begin{array}{c}\text { I } \\
\text { Emocional }\end{array}$ & 0,549 & $* * *$ \\
\hline
\end{tabular}

\section{Conclusiones}

Al realizar el análisis descriptivo de género, se evidenció que las mujeres son las que poseen una mayor participación con emprendimientos de servicios turísticos los datos nos indican que se encuentran en un porcentaje de $(61,6 \%)$ mientras que los hombres $(38.4 \%)$, es decir las mujeres superan en un $23,4 \%$, a los hombres. Los emprendimientos que se han desarrollado en la ciudad de Puyo pertenecen a las personas que han alcanzado estudios secundarios con un porcentaje de $47,8 \%$, y estudios universitarios de $36,5 \%$ esto demuestra que la educación superior no es factor influyente en el momento de realizar un emprendimiento. Los ingresos netos que se obtienen, pertenecen al rango de ganancia del nivel mensual correspondiente de 500 hasta 2000 dólares, que generan los emprendedores después de pagar sus impuestos, materia prima, arriendos y personal, esto concluye que existe una ganancia que le permite mantener su estilo de vida. El análisis multivariante indicó que existe una relación significativa entre la Inteligencia Emocional con la Autoeficacia Emprendedora, es decir la creencia de las habilidades emprendedoras de los propietarios de los servicios turístico de la ciudad de Puyo se encuentra influenciadas por el manejo de la inteligencia emocional, de la misma manera, cada una de las dimensiones de Autoeficacia Emprendedora está determinada por el manejo de la Inteligencia Emocional.

\section{Recomendaciones}

La Inteligencia Emocional de los emprendedores, puede mejorar siempre y cuando exista un posicionamiento estratégico del negocio en el mercado, que permita una estabilidad emocional por mantener ingresos económicos estables, por lo tanto, se puede promover un plan de marketing a nivel local en la ciudad de Puyo, el cual estaría involucrado con los organismos gubernamentales, municipales, académicos y privados para tener un mayor posicionamiento en el mercado y se pueda incrementar el número de clientes. Se recomienda, además, la realización de un conversatorio entre las entidades gubernamentales de turismo y los empresarios de servicios turísticos con el objetivo de conocer y realizar en primer lugar un diagnóstico situacional de los diferentes emprendimientos, con respecto a su presencia en el mercado del Puyo, desarrollo de posibles crecimientos, inversiones, basado en el comportamiento emprendedor con los resultados que se han obtenido en la presente investigación.

\section{Referencias Bibliográficas}

Amorós, J., Etchebarne, S., y Felzensztein, C. (2012). Emprendimiento Internacional en Latinoamerica, desafios para el desarrollo. ESIC, 513-529.

Anderson, J., y Gerbing, D. (1988). Structural Equation Modeling in Practice: A Review and Recommended Two-Step Approach. Psychological Bulletin,, 411423.

Caiza, R., y Molina, E. (2012). Analisis Historico de la Evolucion del turismo en el Terrtorio Ecuatorino. RICIT, 8-24.

Chion, S., y Vincent, C. (2016). Analítica de datos para la modelación estructural. Lima, Perú: Pearson.

Dehter, M. (2010). Vamos el Este. Descargado de http://vamoseleste.com/img/ biblioteca/ser-emprendedorDEHTER .pdf

Duran, P. (2013). Adaptación y validación del cuestionario de inteligencia emocional Trait Meta-Mood Scale 48 (TMMS-48) de Salovey, Mayer, Goldman, Turvey y Palfai (1995), en estudiantes de pregrado de la Facultad de Educación y Humanidades y de la Facultad de Ciencias de la Salud y Alimentos de la Universidad del Bío Bío de la ciudad de Chillán. Descargado de http://repobib.ubiobio.cl/ jspui/handle/123456789/267

Extremera, N., y Fernández, P. (2005). Universidad de Málaga. Descargado de https://www -researchgate.net/profile/Natalio _Extremera/publication/230887045 _Inteligencia_emocional_percibida _y_diferencias_individuales_en_el _meta-conocimiento_de_los_estados _emocionales_Una_revision_de _los_estudios_con_el_TMMS/links/ 00 b 7 d52919feccac 9000

Gessa, A., y Toledano, N. (2011). Turismo, empredimiento y sostenibilidad en los espacios naturales potegidos El caso de Andalucia - España. Scielo, 50-68.

Lado, R., Vivel, M., Otero, L., y Neira, I. (2015). Barreras al mprendimiento hotelero:un analisis sectorial. Red de Revistas Científicas de América Latina y el Caribe, España y Portugal, 86-92.

Lasio, V., Caicedo, G., Ordeñana, X., y Samaniego, A. (2016). Global Entrepreneurship Monitor (Inf. Téc.). Guayaquil.

Maldonado, J., Castro, F., y Corrales, J. (2015). Red de Revistas Científicas de América Latina y el Caribe, España y Portugal. Descarga- 
do de http: / / www.redalyc.org/articulo . oa? id=349851784021

Manosalvas, L. (2017). PUCEP. Descargado de http://tesis.pucp.edu.pe/ repositorio/handle/123456789/8000

Moriano, J., Topa, G., Molero, F., Entenaza, A., y Levy, J. (2012). Autoeficacia para el Liderazgo Emprendedor. Adaptación y Validación de la Escala CESE en España. Red de Revistas Científicas de América Latina y el Caribe, España y Portugal, 171-179.

Organizacion Mundial de Turismo. (2007). OMT. Descargado de http: / / www2 . unwto. org/es

Ortega. (2014). Universidad de Guadalajara. Descargado de https://s3.amazonaws . com/academia.edu. documents/

36021804/Trabajo_Final_EDJOM

_Inteligencia_Emocional_en_las

_Organizaciones.pdf?AWSAccessKeyId= AKIAIWOWYYGZ2Y53UL3A\&Expires=

$1515646389 \&$ Signature=

I ckqMpB 7EDvJeNkPkqVdn3P IqBY\%

3D\&response-content-disposition= inli

Padilla, M., Quispe, A., y Jimenez, W. (2016). Gestion empresarial y emprendimiento con administracion publica para un desarrollo sostenible del turismo. Retos Turisticos, 15(3), 1-11.

Salinas, F., y Osorio, L. (2017). Emprendimientoy EconomíaSocial, oportunidades y efectos enuna sociedad en transformación. CIRIEC-España, Revista de EconomíaPública, Socialy Cooperativa, 129-151.

Salvador, C. M. (2008). Impacto de la inteligencia emocional percibida en la autoeficacia emprendedora. Boletín de psicología(92), 65-80. Descargado de http://dialnet - unirioja.es/servlet/articulo ?codigo=2732147\&orden=169516\&info= link5Cnhttp://dialnet.unirioja.es/ servlet/extart?codigo=2732147

Silva, M., y Alban, J. (2017). Repositorio UTA. Descargado de http://repositorio.uta.edu.ec/ handle/123456789/25389

Recibido: 30 de junio de 2018

Aceptado: 3 de septiembre de 2018 
